\title{
Optimal Assistive Drive System using Mobile Cloud Computing
}

\author{
Sameh A. Salem \\ Department of Electronics, Communications, and Computer Engineering, \\ Faculty of Engineering, Helwan University, \\ Cairo, Egypt
}

\begin{abstract}
No one can deny that mobile devices are increasingly becoming an essential part of our lives, and being used for information delivery, access and communication. In this paper, a novel assistive drive system with mobile offloading is proposed. Three effective measures are integrated for reliable and early drowsiness detection, namely behavioral, vehicle, and physiological measures. These measures give higher quality and relevant information. Additionally, the proposed system uses mobile devices to process readings. However, with huge amount of data and intensive computations, mobiles cannot deliver results in reasonable times. A possible approach is to offload computations onto the cloud.
\end{abstract}

\section{Keywords}

Mobile Cloud Computing, Computational Offloading, Energy Preserving, Fatigue detection, Computer vision.

\section{INTRODUCTION}

Road traffic injuries are currently the ninth leading source of death globally, and essential reason of death for youth people between the ages of 15-29. Existing trends propose that by 2030 road traffic deaths will turn into the fifth leading cause of death unless crucial action is taken [1]. Latest statistics shows that worldwide the total number of road deaths remains intolerably high at 1.27 million per year [1]. Only twenty-eight countries, covering $7 \%$ of the world's population, have inclusive road safety laws on five key risk factors: speeding and seat-belts failing to use motorcycle helmets, driving and drinking, and child restraints. Driver fatigue and distraction is a considerable factor in a large number of vehicle accidents. Moreover, driving over a large period causes immoderate fatigue and tiredness which makes the driver sleepy or loose awareness and fail to make any maneuvers to avoid a crash. As reported in [2], the number of hours consumed in driving has a strong correlation to the number of fatigue related accidents. Fig. 1 demonstrates the relationship between number of hours driven and the count of crashes in percent with relation to driver fatigue [2]. A driver who dosses at the wheel loses control of the vehicle, a disposal which often results in a crash with either another vehicle or stationary objects. In order to block these destructive accidents, the state of drowsiness of the driver should be observed [3]. The aim of this paper is to use the recent advances in mobile cloud computing which provides unlimited computing resources to develop a reliable and accurate fatigue detecting system which uses nonintrusive measures. The paper is organized as follows: Section 2 shows mobile cloud computing architectures and services. Section 3 introduces the proposed assistive drive system that includes reliable fatigue detection system integrated with mobile cloud computing. Finally, Section 4 and 5 present the discussions and conclusions respectively.

Percentages of crashes due to fatigue as a function of hours of driving

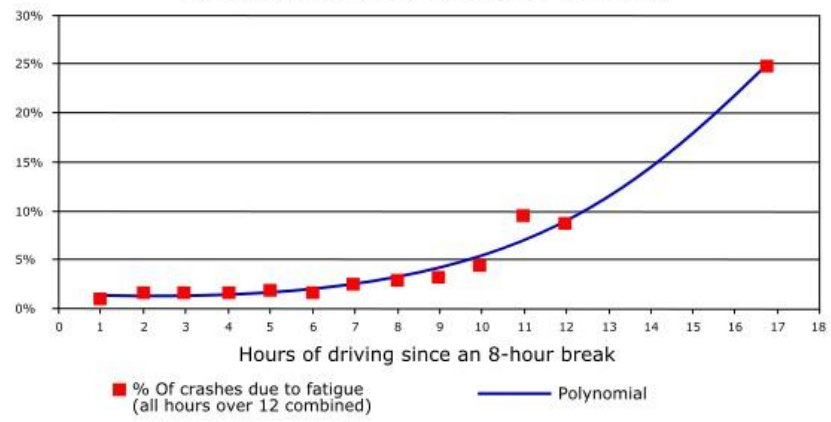

Fig. 1: The relationship between number of hours driven and the number of crashes in percent for driver fatigue [2]

\section{CLOUD COMPUTING}

Cloud computing is a new technology that aims to efficiently provide pervasive, convenient, and on-demand access to resources [4-7]. It could be considered as an unlimited resource that can be obtained anytime and anywhere in the world. It supplies computing, storage, services, and applications over the Internet. Moreover, cloud computing ease to reduce capital cost, separate services from the underlying technology, and gives flexibility in terms of resource supplying.

Since mobile devices are increasingly becoming an essential part of human life, it still lack in resources compared to a conventional information processing device such as PCs and laptops. Therefore, mobile cloud application models are designed to fulfill a particular objective, such as executing applications that have inadequate resources for local execution, enhancing applications performance (in terms of computation time), or obtaining energy efficiency on mobile devices [5, 6, 8] Therefore, Mobile Cloud Computing (MCC) is the consequence of interdisciplinary approaches comprising mobile computing and cloud computing. This will allow the possibility of making fatigue detection systems more affordable and portable. According to a recent research [7], mobile cloud computing will indeed start to govern the industry. 


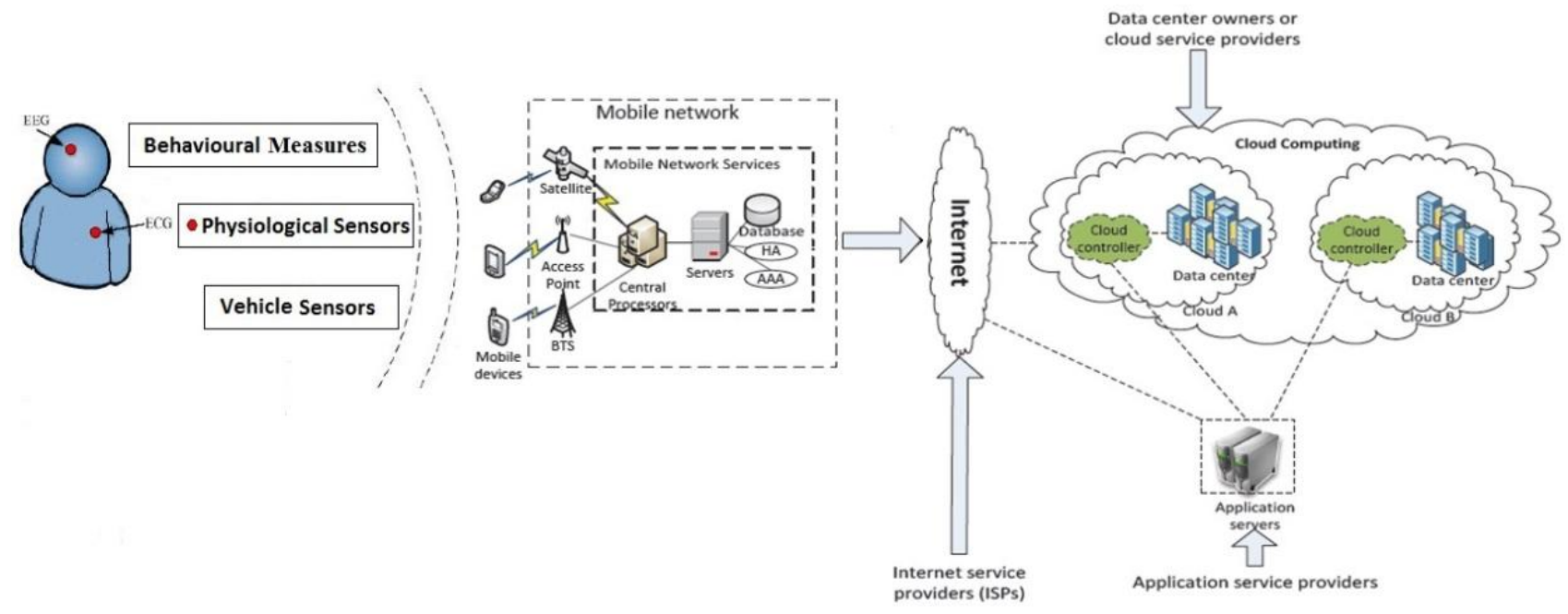

Fig. 2: Proposed architecture of the Assistive Drive System using Mobile Cloud Computing

Computation offloading is a process that migrates very huge computations from a mobile device to the unlimited resources of cloud, or server (called nearby infrastructure). Offloading the heavy computations to the cloud decreases the power consumption of mobile batteries, improves the applications performance, and executes mobile applications that are unable to execute due to insufficient resources [9-12]. Furthermore, cloud gives storage services that could be used to overwhelm the storage limitations of the mobile devices. In this context, road safety and fatigue detection are one of the applications that can benefit from the combination of mobile and cloud technologies.

\section{PROPOSED SYSTEM}

The architecture of the introduced system is shown in Fig. 2. It is composed of three main components: non-intrusive sensors for collecting behavioral, physiological, and vehicle signals, mobile devices for gathering, processing, and updating sensor readings, and cloud servers which provide computing resources along with database servers, and data mining servers. As shown, mobile devices are linked to the mobile networks via base stations (e.g., access point, satellite, or base transceiver station (BTS) that set up and monitor the connections and interfaces between the mobile devices and networks [13]. Mobile device's information, such as ID and location, are transferred to the central processors that are linked to servers giving mobile network services. Here, the network operators for mobile services can extend facilities to mobile devices as AAA (for authorization, authentication, and accounting) based on home agent (HA) and the data is saved in databases. After that, mobile device's information is transmitted over the internet to the cloud. The cloud controllers manipulate the information and assign the necessary computing resources. Then provide mobile devices with the necessary instructions along with alert and warning signals based on configurable setting.

\section{Fatigue detection}

Three effective measures are integrated for reliable and early detection of drowsiness, namely behavioural, vehicle, and physiological measures. Vision subsystem, as a behavioural measure, is introduced to monitor driver's eye for detecting fatigue and distraction. Additionally, physiological sensors are used to provide a significant change in earlier stages of drowsiness that gives the possibility of predicating the drowsiness and warning in a timely basis the drowsiness drivers. While, vehicle sensors are used to detect the steer wheel movements and the closest objects around the vehicle and road barriers.

Vision subsystem: The most important aspect of implementing a machine vision system is the image acquisition [14]. Any deficiencies in the acquired images can cause problems with image analysis and interpretation. Examples of such problems are a shortage of detail due to insufficient dissimilarity or poor positioning of the camera: this can give rise to the objects be unrecognizable, so the purpose of vision cannot be fulfilled. In this subsystem, a driver camera is being used to monitor driver's eye for detecting fatigue and distraction. A detection system for drowsy drivers is introduced through the use of non-intrusive vision concepts. The system uses smartphone camera to point directly towards the driver's face and monitors the eye of the driver in order to detect fatigue. In such a case when fatigue is discovered, a warning signal is determined to notify the driver. This subsection demonstrates how to identify the eyes, and how to obtain whether the eyes are open or closed. The system deals with using information obtained for the binary version of the image and apply HAAR cascaded classifier $[15,16]$ to detect eyes. Upon a time the eyes are identified, a series of algorithms can be applied to mark whether the eyes are open or closed. Experimentally, if the eyes are found closed for five sequential frames, the system draws the conclusion that the driver is falling asleep and releases a warning signal. The system is also capable of discover when the eyes cannot be found, and works under logical lighting conditions.

In this paper, robust and fast real-time system for face detection is used [15]. It requires minimum computational time while giving higher accuracies of detection along with low positive rates These promise its usage on small and low power devices including mobile devices, hand-held, and embedded processors. Additionally, it operates with wide range of conditions including illumination, scale, pose, camera variation. The minimum/low computational time is achieved through the use of integral image representation [15] that can be computed using a few operations per pixel for an image. . The high detection accuracy is achieved through the use of cascaded classifier which is applied on a small number of important features, called Haar-like features that are selected by AdaBoost [15, 16]. Fig. 3a, illustrates Haar-like features. In this context, instead of using the intensity value of a pixel, one can use the variation in contrast values among adjacent rectangular groups of pixels. These contrast variances mark the relative light and dark regions that give identical properties of the faces (e.g., the eyes area is dusky compared with the upper- 
cheeks and the nose bridge area is brighter than the eyes). Furthermore, each rectangle feature can be examined at any scale and location. Fig. 3a depicts the performance evaluation by using Receiver Operating Characteristic Curve (ROC). As shown, higher true detection accuracies with low positive rates are achieved. It should be noted that the face detection phase

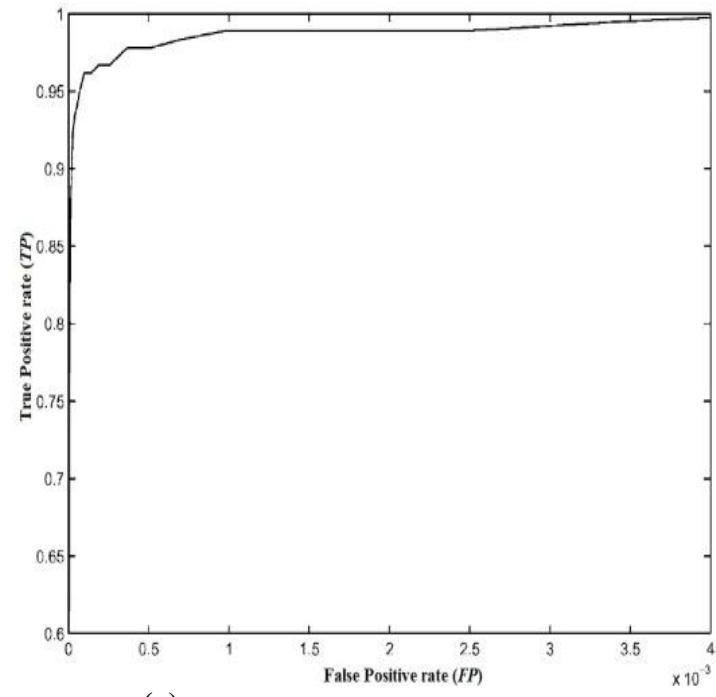

(a) consumes a fraction of seconds for face detection, which highly meet the requirements of the proposed system. Once the face is detected in the frame, the search area for extracting the eyes is limited to make the computation process much faster. The eyes are found in the upper most part of the face.

Fig. 3: (a) Common Haar Features, and (b) Performance evaluation using ROC curve

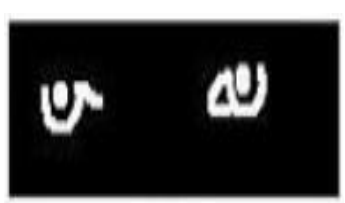

a) Canny detector

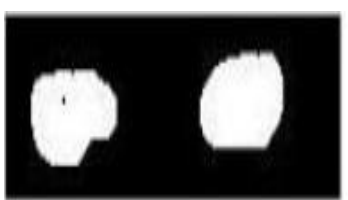

(b) Dilation
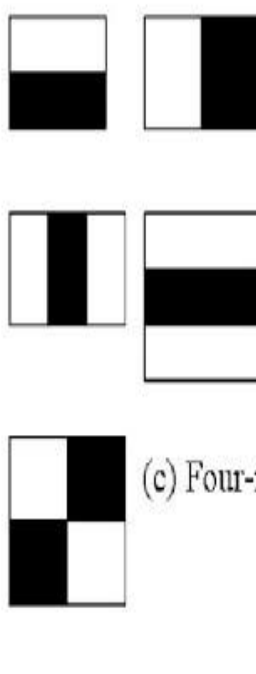

(a) Edge Features

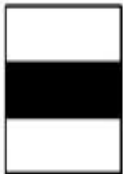

(b) Line Features

(c) Four-rectangle features

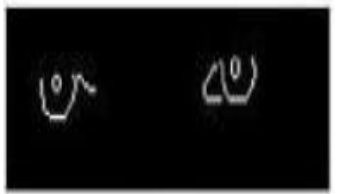

(c) Hough Transform

Fig. 4: Performance evaluation using ROC curve

Canny edge detection $[14,17]$ is applied on the selected area for the eyes as depicted in Fig. 4a. Subsequently morphological dilation $[14,18,19]$ is performed to make the edge image more prominent for further processing as shown is Fig. 4b, Circular Hough transform is applied to the dilated image to locate the circle. Fig. $4 \mathrm{c}$ shows the resultant image. Coordinates of the right and left eye are acquired by using feature measurement as minor axis length, eccentricity convex area, major axis length etc., on the transformed image. After this step, it becomes easy to determine whether the eyes are close or opened. Experimentally, if the eyes are found closed for five sequential frames, the system draws the illation that the driver is falling asleep and issues a warning signal.

It should be noted that all computer vision algorithms were developed using OpenCV (Open source Computer Vision Library: <http://opencv.org>). It has $\mathrm{C}, \mathrm{C}++$, JAVA interfaces and Python and supports android, iOS, Linux, Windows, and Mac OS. OpenCV was designed for computational qualification and with a robust concentrate on real-time applications [19]. It was developed, released under a BSD license, and freely obtainable for both freely commercial and academic use.

Vehicle Sensors: Two kinds of sensors are considered. The first is the sensors that are used to detect the road barriers and the closest objects, while the second sensor is the Steering Wheel Movement (SWM) which is used for revealing the level of driver drowsiness. For the first type of sensors, the system can monitor the source of barriers and their distances with configurable contrasts. In fact, there are many sensors to determine the distances as ultrasonic sensors, infrared sensors, laser rangefinders, radar and sonar. In this paper, ultrasonic sensors are used. It gives a reliable and low cost source for detecting obstacles. Since it is not vision-based, it is beneficial under conditions of indigent lighting and transparent objects. The ultrasonic sensor transmits a brief chirp with its ultrasonic speaker and makes it conceivable for the basic stamp to measure the time it takes the echo to return to its ultrasonic microphone.

In this paper, Parallax Ping sensor is used [20]. It identifies objects by producing a small ultrasonic burst and then waiting for an echo. A host processor or microcontroller gives a pulse trigger to enable the sensor to output an ultrasonic burst of $40 \mathrm{kHz}$. This burst propagates for about 1130 feet per second, meets an object and then rebound to the sensor. The sensor gives an output signal to the host that will stop when the echo is identified. In this context, the pulse width matches to the object distance. Additionally, it is easily noted the accurate detection of an object distance close to the half centimeter to 3.3 meters. On the basis of the distances of the objects, two alerting signals can be produced to the driver once an object is detected. The first is a warning signal with white sign which means safe distance, whereas the other signal is a warning and vibrating signal with red sign which means unsafe distance. This unsafe distance is pre-configured by the owner of the vehicle. It should also be noted that the proposed system does not rely on object distances only but also on the vehicle speed. For example, when the driver is driving at 80 $\mathrm{Km} / \mathrm{H}$, the vehicle should have about two meters ahead in case of sudden moves or actions. 
For the second type of sensors, a steering angle sensor that could be used as a measure for identifying the driver drowsiness's level achieves Steering Wheel Movement (SWM). By using an angle sensor attached on the steering column, the driver's steering behavior can be measured. At drowsiness state, the steering wheel micro-corrections count is decreased compared to the normal state of driving. In [3], the sleep and drowsy drivers performed lower reversals for the steering wheel than normal drivers did. To avoid the lane changes effect, the researchers deemed only few steering wheel movements (between $0.5^{\circ}$ and $5^{\circ}$ ), which are essential to adapt the lateral position within the road [21]. Hence, based on small SWMs, it is used to alert the driver at the first signs of sleepiness, and that is used as an early indicator of the drowsiness.

Physiological Sensors: Although the reliability of the proposed vision subsystem and vehicle sensors in early detection of drowsiness. It discovered after sleep starts, which might be late for avoiding an accident. However, physiological signals such as electrocardiogram (ECG), electromyogram (EMG), electrooculogram (EOG) and electroencephalogram (EEG) give the real internal state of the driver as it is starting to change in earlier stages of drowsiness [3, 22]. Fig. 5 shows an instance of brain wave activity for different sleep phases. As shown, there is dissimilarity between the brain wave for awake and the brain waves of distinct sleep stages. Studies [23, 24] show that ECG will clearly decrease when a driver is exhausted and the changes of the heart rate have a potential relationship to the degree of drowsiness of the driver. Therefore, physiological signals are reliable, accurate and more appropriate to identify drowsiness; enabling the alert of a drowsy driver in a reasonable time and thereby prevents numerous road accidents. However, the high intrusive nature of the physiological sensors makes it unrealistic since the electrodes would have to be directly connected to the driver which will distract the driver and hinder the driving.

Recently, there is an increase of non-intrusive sensors [25, 26], which enable the use of physiological sensors in detecting the driver fatigue. Therefore, it is possible to attach wireless noncontact electrodes on the steering wheel or on the driver's seat. It should be noted that the non-intrusive system accuracy is relatively less due to the errors and artifacts of the movement that happen because of the improper contact of the electrodes. Therefore, for reliable readings, there is a recently developed wearable ECG acquisition device [26]. This device uses dry foam polymer based electrodes that do not need conduction gels and can give good conductivity to get ECG signal effectively, and can adapt to irregular skin surface to maintain low skin-electrode impedance and reduce motion artifacts under movement. This could be built as driving suit.

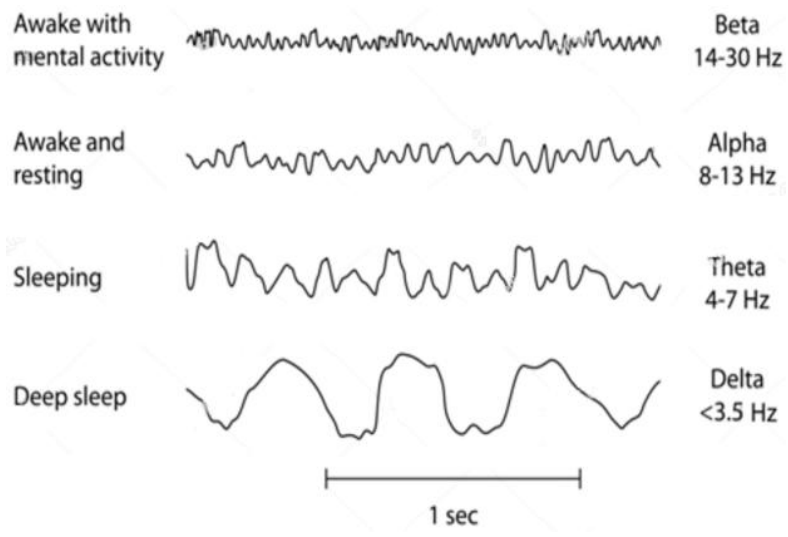

Fig. 5: An example of brain wave activity for different sleep stages (Source: http://www.shutterstock.com/)

\subsection{Cloud Offloading}

Since mobile devices and smartphones are increasingly becoming an essential part of human life, the proposed Assistive Drive system is tested using well-known and commonly used smartphones using various data service connections, Samsung Galaxy Note and iPhone. Fig. 6 shows the power in percentage of smartphones batteries when running the proposed Assistive Drive System. As shown, the power consumption falls to about $26 \%$ and $12 \%$ in three hours for Samsung Galaxy and iPhone respectively. Therefore, there is a need for alternative strategies to save the power consumption and quickly process different sensor readings; computation offloading is used to deliver processing results in a reasonable time and save the power consumption. This gives advantageous to the proposed system to be more affordable and portable. In this context, immigrating large computations and complex processing from resourcelimited devices to resourceful machines (i.e., servers in clouds) that have efficient parallel computing abilities can be advantageous. This avoids late responses for drowsiness detection because of long execution times on mobile devices. It should be noted that the computation is done either by offloading a task completely or partially to the cloud. This is achieved either statically or dynamically. For static environments, the offloading is determined by a tradeoff between communication and computation cost, while for dynamic environments, the offloading is determined by the connection status and bandwidth. In this paper, dynamic environment is convenient for fatigue detection. Fig. 7 shows the basic workflow of the computation offloading process. The workflow begins with executing an application pursued by examining the user's offloading permission. If offloading is allowed, then the application examines the cloud connectivity and obtains the available/assigned resources. Then the followed step includes the offloading decision. If the offloading is preferable, then the computation offloading is achieved. Otherwise, the application performs locally the entire computations.

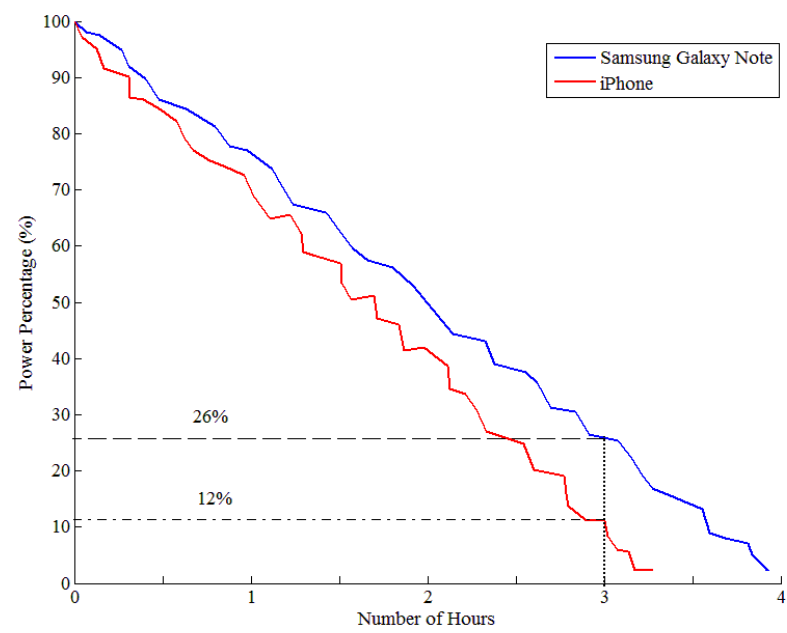

Fig. 6: Power consumptions of Samsung Galaxy \& iPhone when running the proposed Assistive Drive System

For this purpose, CloneCloud [10] is adopted in this paper. It is based on an augmented approach that offloads segments of application execution to the adjacent infrastructure or cloud. Therefore, when augmentation is required, the smartphone application process enters a sleep state and transfers the process state to the clone. The virtual machine creates a new process state and overlays the received information, followed by execution of the clone. On completion of the execution, the state of the process for the clones' application is transmitted to the 
smartphone, where the process state is recombined into the smartphones' application and the application comes out of a sleep state. Fig. 8 demonstrates the CloneCloud offloading process. For this purpose, several experiments have been carried out to examine the effect of applying CloneCloud offloading to the proposed system on Samsung Galaxy Note smartphone having Android operating system, $1.4 \mathrm{GHz}$ dual core Cortex-A9 CPU, 1 GB RAM, 3G and WiFi connections. It is worth to note that the clones encompassed are tailored AMIs (Amazon Machine Image) of Android-x86 OS and executed at Amazon EC2 platform. Two evaluation parameters have been used. The first is the Speedup parameter which is the ratio between the execution time when running the application with CloneCloud to the execution time when running on phone only. While the second parameter is the energy consumption relative to the phone. In these experiments, $3 \mathrm{G}$ data connectivity and $\mathrm{WiFi}$ access are considered. As shown in Fig. 9, CloneCloud with 3G data service connectivity achieves about $11 \%$ speedup with $44 \%$ energy consumption compared with running the application on phone only. For CloneCloud with $\mathrm{WiFi}$ access on roadsides, it achieves over $20 \%$ speedup with about $23 \%$ energy consumption relative to phone consumption. These results indicate the CloneCloud offloading improves not only the computational speed but also saves the phone battery. Therefore, three objectives are achieved through cloud offloading. The first is enhancing the performance of the proposed Assistive Drive system application on mobile devices. Secondly, achieve energy efficiency application. Finally, it makes fatigue detection systems more affordable and portable.

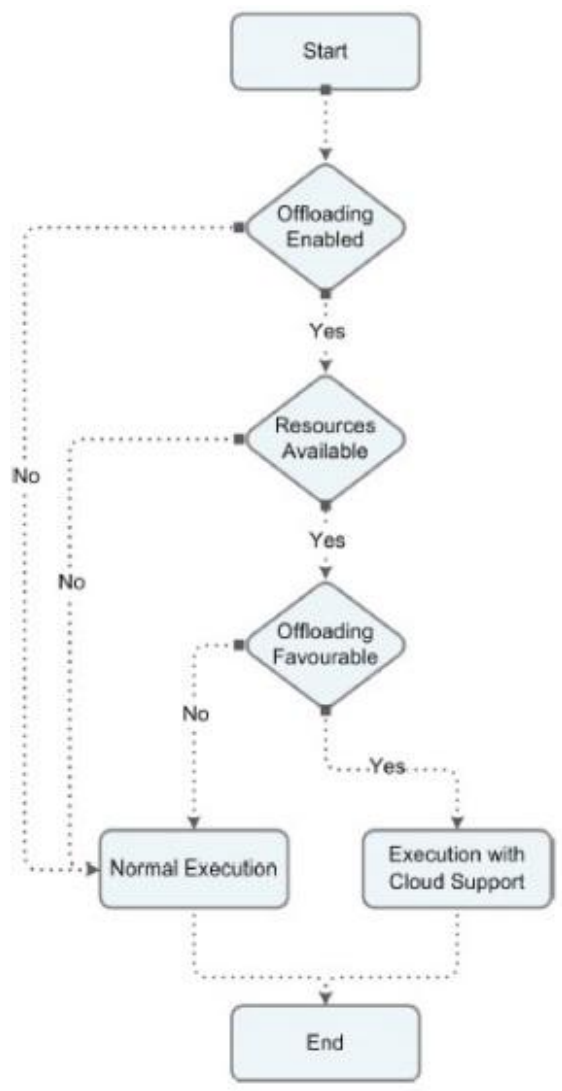

Fig. 7: Computational offloading process,

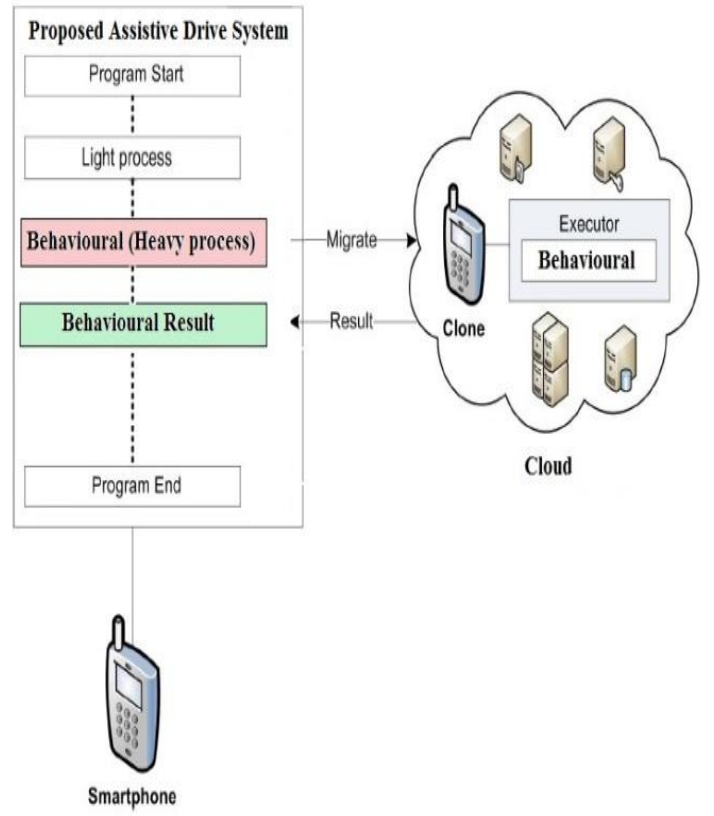

Fig. 8: Assistive Drive application with CloneCloud

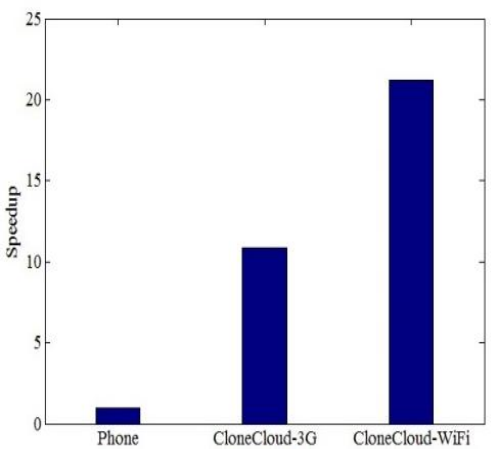

(a) Speedup

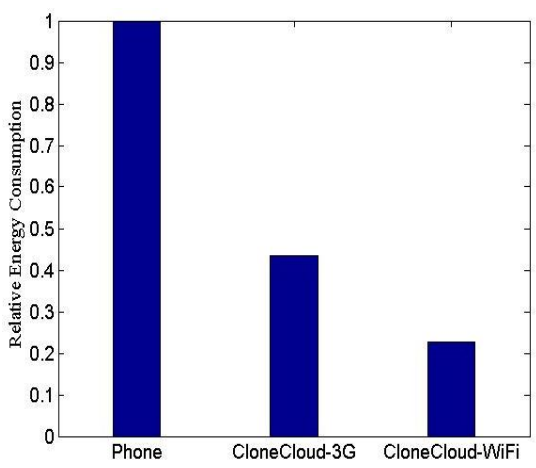

(b) Relative Energy Consumption

Fig. 9: Performance Evaluation

\section{DISCUSSIONS}

In fact, each measure used for discovering drowsiness has its own limitations and advantages. However, with increasing availability of non-intrusive sensors, it becomes possible and beneficial to combine behavioral, vehicle, and physiological measures. Therefore, by integrating these independent measures provide us with a reliable drowsiness indicator which reduces the chance of false detection. Furthermore, with the use of mobile cloud computing which comprises the mobile computing and cloud computing, it allows the possibility of making fatigue 
detection systems more affordable and portable. Consequently, any mobile device ranging from feature phones to smartphones can be used. To the best of our knowledge, this is the first work that uses the mobile cloud computing in fatigue detection systems.

In fact, early predication of driver fatigue can add value to Vehicular communication systems in which vehicles are the communicating nodes, providing each other with information, such as safety warnings, traffic information, and fatigue status of the nearby vehicles. As a collaborative approach, vehicular communication systems can be better efficient in traffic congestions and avoiding accidents than if each vehicle attempts to resolve these problems individually.

Additionally, transferring fatigue status in a centralized fashion can give us a significant improvement in estimating fair car insurance. Therefore, no one can get cheap car insurance if he/she have little experience or have higher fatigue scores. Therefore, it is perhaps not surprising that car owners can pay more than double the average car insurance premium. Furthermore, penalty points can be added to the driving licenses after certain level of fatigue in addition to driving offenses. Consequently, if the total exceeds a specified limit the driver may be disqualified from driving for a time, or the driving license may be revoked.

\section{CONCLUSIONS}

In this paper, a novel assistive drive system using mobile cloud computing has been proposed. As demonstrated, mobile cloud computing, which combines the strength of clouds and the convenience of mobile terminals, provides a promising model. Experimental results showed the significant effect of cloud offloading in energy saving and computational speed. The proposed system uses the availability of unlimited resources of cloud for processing sensor readings in a reasonable time. Three robust measures are combined and have been used for early detection of driver fatigue. This combination results in a crucially higher success rate than any individual measure. As demonstrated, non-intrusive sensors have been used to avoid any distractions that might hinder the driving. With our detection system, acute and deemed drowsy can be early discovered in time and can send out automated alerts before a mishap happens. We believe that it is not safe and ethical to leave a drowsy driver drive on road.

\section{REFERENCES}

[1] "World Health Organization". Global status report on road safety 2017. World Health Organization, 2017.

[2] "Regulatory impact and small business analysis for hours of service options," Technical report, Federal Motor Carrier Safety Administration, February 2011.

[3] H. Abbood, W. Al-Nuaimy, A. Al-Ataby, S. A. Salem, and H. S. Alzubi, "Prediction of driver fatigue: Approaches and open challenges," 2014 14th UK Workshop on Computational Intelligence (UKCI), 2014.

[4] R. Buyya, J. Broberg, and Gościński Andrzej, Cloud computing: principles and paradigms. Hoboken, NJ: Wiley, 2011 .

[5] R. Sosan and C. F. Azim, "RETRACTED ARTICLE: Mobile Cloud Computing: The Taxonomy and Comparison of Mobile Cloud Computing Application Models," Wireless Personal Communications, vol. 89, no. 4, pp. 1435-1435, 2016.

[6] A. U. R. Khan, M. Othman, S. A. Madani, and S. U. Khan,
"A Survey of Mobile Cloud Computing Application Models," IEEE Communications Surveys \& Tutorials, vol. 16, no. 1, pp. 393-413, 2014.

[7] A. Priyanka, "Mobile cloud computing." International Journal of Engineering and Advanced Technology (IJEAT) vol. 2, pp. 606-609, 2013.

[8] S. Abolfazli, Z. Sanaei, M. Alizadeh, A. Gani, and F. Xia, "An experimental analysis on cloud-based mobile augmentation in mobile cloud computing," IEEE Transactions on Consumer Electronics, vol. 60, no. 1, pp. 146-154, 2014

[9] W. Zhang, Y. Wen, "Energy-Efficient Task Execution for Application as a General Topology in Mobile Cloud Computing," IEEE Transactions on Cloud Computing, vol. 6, issue 3, pp. 708-719, 2018.

[10] B.-G. Chun, S. Ihm, P. Maniatis, M. Naik, and A. Patti, "Clonecloud: Elastic execution between mobile device and cloud" Proceedings of the sixth conference on Computer systems - EuroSys 11, 2011.

[11] M. V. Barbera, S. Kosta, A. Mei, and J. Stefa, "To offload or not to offload? The bandwidth and energy costs of mobile cloud computing," 2013 Proceedings IEEE INFOCOM, 2013.

[12] S. A. Said, S. A. Salem, S. G. Sayed, "Energy Aware Mobile Cloud Computing Algorithm for Android Smartphones," In Proceedings of the International Conference on Advanced Intelligent Systems and Informatics 2017, AISI2017, Advances in Intelligent Systems and Computing, vol 639. Springer, Cham, 2018.

[13] E. R. Davies, Machine vision theory, algorithms, practicalities. San Francisco, CA, USA: Elsevier, 2012

[14] P. Viola and M. Jones, "Rapid object detection using a boosted cascade of simple features," Proceedings of the 2001 IEEE Computer Society Conference on Computer Vision and Pattern Recognition. CVPR 2001.

[15] J. Zhu, Z. Chen, "Real Time Face Detection System Using Adaboost and Haar-like Features," In $2^{\text {nd }}$ IEEE International conference on Information Science and Control Engineering, pp. 404-407, 2015.

[16] Juseong Lee, Hoyoung Tang, Jongsun Park, "Energy Efficient Canny Edge Detector for Advanced Mobile Vision Applications", IEEE Transactions on Circuits and Systems for Video Technology, vol, 28, issue 4, pp. 1037 1048, 2018.

[17] Anamika Singh ; Manminder Singh ; Birmohan Singh, " Face detection and eyes extraction using sobel edge detection and morphological operations" IEEE International conference on Advances in Signal Processing (CASP), pp. 295-300, 2016.

[18] R. C. Gonzalez and R. E. Woods, Digital image processing. Upper Saddle River, NJ, USA: Prentice-Hall, Inc. 2006.

[19] R. Laganiere, OpenCV 3 Computer Vision Application Programming Cookbook. Birmingham: Packt Pub., 2017.

[20] ParallaxInc.[Online].Available:https://www.parallax.com/p roduct/28015. [Accessed: 15-Oct-2018]

[21] S. Otmani, T. Pebayle, J. Roge, and A. Muzet, "Effect of driving duration and partial sleep deprivation on subsequent alertness and performance of car drivers," Physiology \& 
Behavior, vol. 84, no. 5, pp. 715-724, 2005.

[22] B.-G. Lee, S.-J. Jung, and W.-Y. Chung, "Real-time physiological and vision monitoring of vehicle driver for non-intrusive drowsiness detection," IET Communications, vol. 5, no. 17, pp. 2461-2469, 2011.

[23] V. P. Nambiar, M. Khalil-Hani, C. Sia, and M. N. Marsono, "Evolvable Block-based Neural Networks for classification of driver drowsiness based on heart rate variability," 2012 IEEE International Conference on Circuits and Systems (ICCAS), 2012.

[24] G. D. Furman, A. Baharav, C. Cahan, and S. Akselrod, "Early detection of falling asleep at the wheel: A Heart
Rate Variability approach,” 2008 Computers in Cardiology, 2008.

[25] B.-S. Lin, W. Chou, H.-Y. Wang, Y.-J. Huang, and J.-S. Pan, "Development of Novel Non-Contact Electrodes for Mobile Electrocardiogram Monitoring System," IEEE Journal of Translational Engineering in Health and Medicine, vol. 1, pp. 1-8, 2013.

[26] I.-J. Wang, L.-D. Liao, Y.-T. Wang, C.-Y. Chen, B.-S. Lin, S.-W. Lu, and C.-T. Lin, "A Wearable Mobile Electrocardiogram measurement device with novel dry polymer-based electrodes," TENCON 2010 - 2010 IEEE Region 10 Conference, 2010. 